\title{
A tilted angle polarization type piezoelectric transducer for plate wave generation
}

\author{
Kazuhiko Imano ${ }^{\text {a) }}$ \\ Department of Electrical and Electronic Engineering, Faculty of Engineering and \\ Resource Science, Akita University, 1-1 Tegata Gakuen-cho Akita 010-8502, Japan \\ a)imano@ipc.akita-u.ac.jp
}

\begin{abstract}
A tilted angle polarization type piezoelectric transducer (TAPP) is newly suggested to generate the Lamb waves in thin plate without wedge. The tilted angle between the TAPP and the aluminum plate is easily determined by Snell's law. Our new type TAPP with a tilted angle of $50^{\circ}$ for S0 mode Lamb wave generation was specially manufactured. Preliminary experiments have been performed to examine the usefulness of our TAPP. As a result, efficient radiation of S0 mode Lamb wave from TAPP was confirmed and was clearly identified by the wavelet transform analysis.
\end{abstract}

Keywords: polarization, tilted angle, plate wave, Lamb wave

Classification: New functional devices and materials

\section{References}

[1] J. L. Rose, Ultrasonic Waves in Solid Media, Cambridge University press, Cambridge, 2004.

[2] Fuji Ceramics Co. Ltd., [Online] http://www.fujicera.co.jp/product/e/ index.html.

[3] M. Z. Silva, R. Gouyon, and F. Lepoutre, "Hidden corrosion detection in aircraft aluminum structures using laser ultrasonics and wavelet transform signal analysis," Ultrasonics, vol. 41, no. 4, pp. 301-305, 2003.

[4] H. Horie, M. Nishihira, and K. Imano, "Real time-measurement of acoustic properties of coating material using surface wave and plate wave," Jpn. J. Appl. Phys., vol. 44, no. 6B, pp. 4333-4335, 2005.

[5] M. Watanabe, M. Nishihira, and K. Imano, "Detection of defects on reverse side of metal plate using $\mathrm{MHz}$ range air-coupled Lamb wave," Jpn. J. Appl. Phys., vol. 45, no. 5B, pp. 4565-4568, 2006.

\section{Introduction}

The Lamb wave is one kind of plate wave which propagates in a thin plate. The unique features of Lamb waves are lossless propagation and the existence of various propagation modes in plate like materials. The application of Lamb waves is thus expected as an important method in the field of nondestructive 
inspection (NDT) [1]. For the generation of a Lamb wave, a wedge has usually been used between the piezoelectric transducer and the plate being tested. The wedge operates as the converter from a bulk wave to a plate wave. The wedge must be fixed at the plate using a coupling fluid such as oil or grease; hence, high speed scanning is not practical.

In this paper, our new tilted angle polarization type of piezoelectric transducer (TAPP) is introduced for generation of the Lamb waves in thin plates. Preliminary experiments have been carried out to indicate the usefulness of this new transducer.

\section{Tilted angle polarization type of piezoelectric transducer}

Usually plate type piezoelectric transducers are polarized normal or parallel to their surfaces so that the vibration directions are mainly dominated by these polarization directions. It is therefore expected that the vibration direction could be controlled by tilting the polarization direction. In accord this idea, TAPPs were prepared to confirm the possibility of tilting the sound radiation direction. TAPPs manufactured by Fuji Ceramics Co. Ltd.: C-13 [2] have the tilted angle $\theta_{c 1}$ of $50^{\circ}$ as shown in Fig. 1. The tilted angle $\theta_{c 1}\left(=50^{\circ}\right)$ was designed to the critical angle between the transducer and aluminum plate by Snell's law to generate a S0 mode Lamb wave. Rectangular $(20 \mathrm{mmW} \times 40 \mathrm{mmL} \times 1 \mathrm{mmt})$ type piezoceramic plates were specially manufactured. The resonant frequency of both normal type and TAPP transducers, $\left(0^{\circ}\right.$ and $\left.50^{\circ}\right)$ were $1 \mathrm{MHz}$.

\section{Experiments}

The experimental system is shown in Fig. 1. Both TAPP and normal PZT transducers were used as transmitters and the usual wedge type transducer was used as a receiver.

The distance between transmitter and receiver was set to $200 \mathrm{~mm}$ and $1 \mathrm{~mm}$ thick aluminum plate was used as the plate sample. Transmitters were driven by $1 \mathrm{MHz}$ sine-burst voltage of $10 \mathrm{VP}$-P.

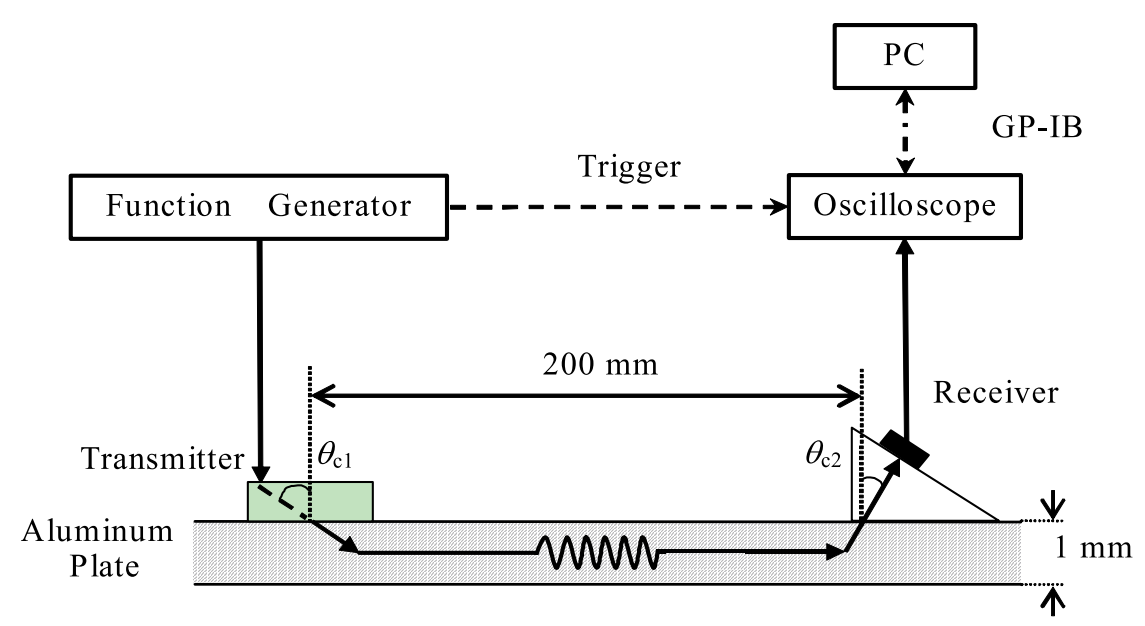

Fig. 1. Experimental system 
Fig. $2(\mathrm{a})$, (b) shows the received waveform transmitted by the normal transducer and TAPP, respectively. Using TAPP, a received amplitude of more than $10 \mathrm{~dB}$ compared to that obtained by a normal piezoelectric transducer was accomplished. To examine the mode of the received waves, wavelet transform was performed $[3,4,5]$. Fig. 3 shows the time-frequency diagram that superposes the dispersion curves of S0 (solid line) and A1 (dotted line) of a Lamb wave. In both figures, the shaded areas correspond to the concentration of energy of an ultrasonic wave. The S0 mode Lamb wave is clearly generated at $1 \mathrm{MHz}$ by TAPP while several dispersed modes are generated separately with a normal type transducer.

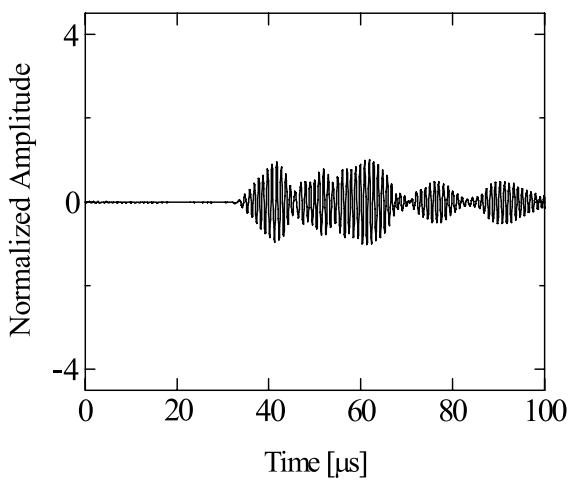

(a)

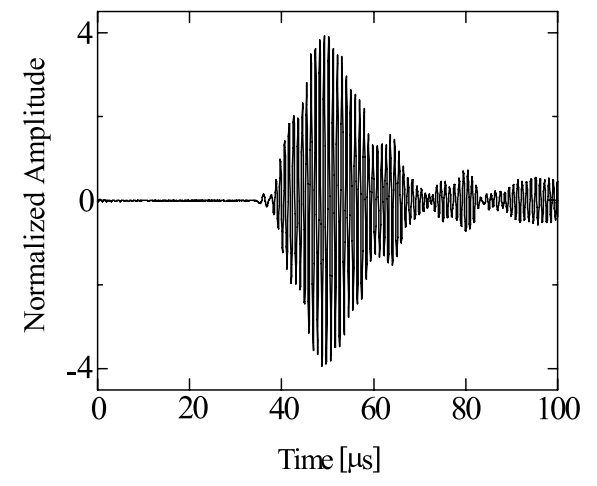

(b)

Fig. 2. Received waveforms. Normal piezoelectric transducer (a) and TAPP (b)

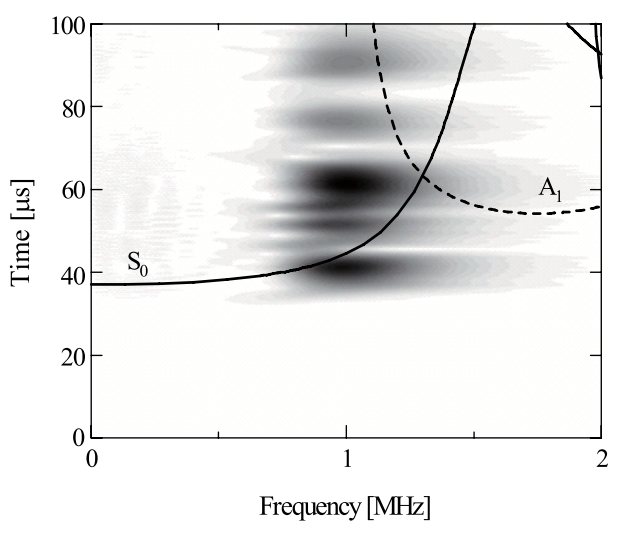

(a)

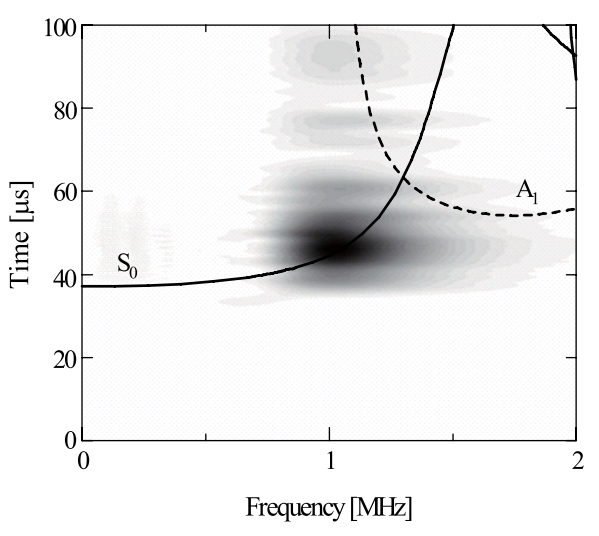

(b)

Fig. 3. Time-Frequency diagram of Fig. 2 (a), (b), respectively

\section{Conclusion}

Our TAPP transducer, a tilted angle polarization type piezoelectric transducer, is proposed for wedge free generation of Lamb waves in thin aluminum plates. In preliminary experiments, we successfully generated the desired 
mode (S0 mode) with high efficiency using the TAPP. TAPP can directly radiate a Lamb wave in the plate sample; therefore, an inspection using high power ultrasound including nonlinear effects will be possible.

Future work is the detailed examination of relationship between generation efficiency and tilted angle. 